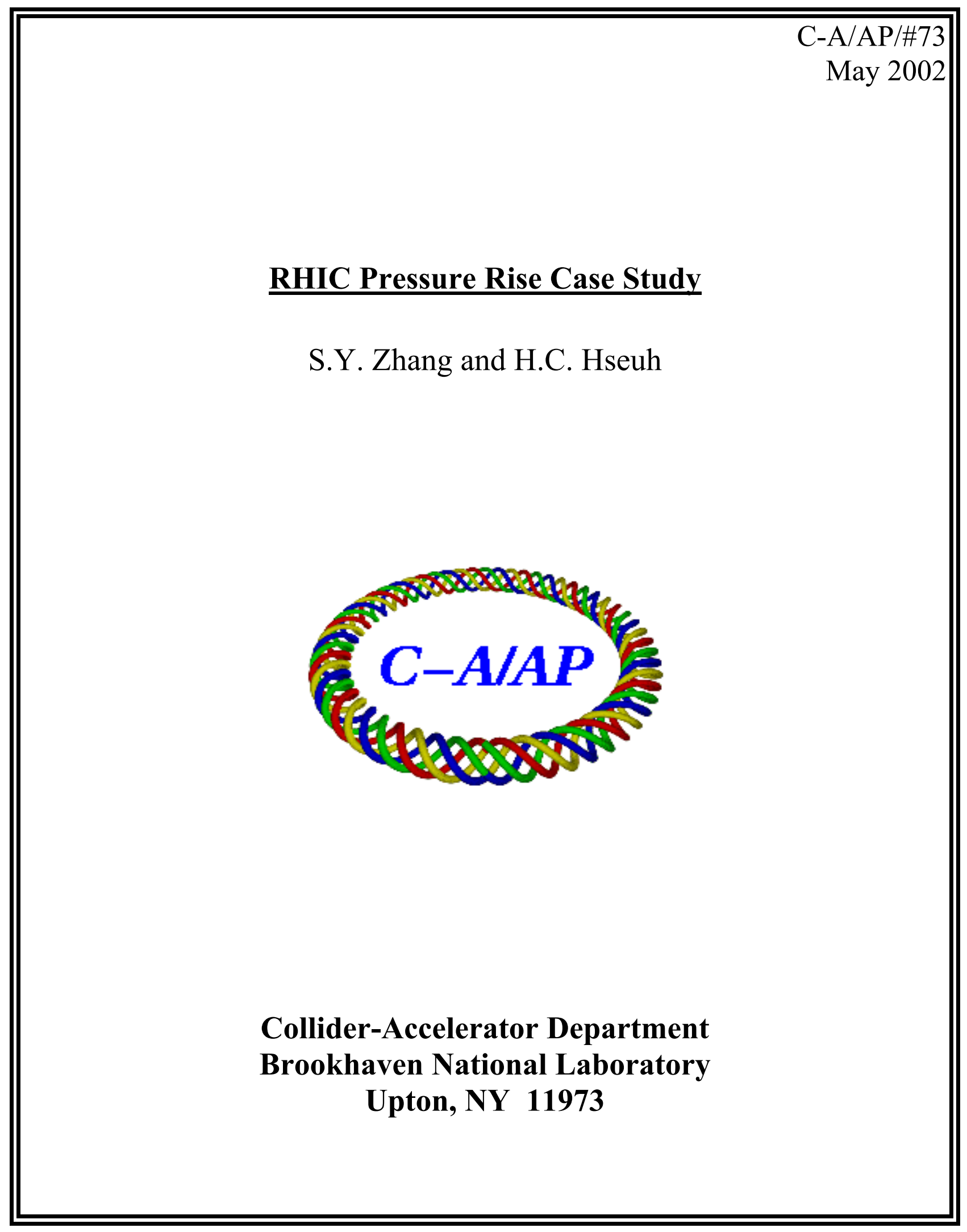




\title{
RHIC Pressure Rise Case Study
}

\author{
S.Y. Zhang and H.C. Hseuh
}

\begin{abstract}
The pressure rise cases of last RHIC run are studied, some observations and analyses are presented. Given limited cases, conclusive results have to wait until next run. Our goal is to get some consensus on the pressure rise issues, which will be useful in planning next luminosity run and study. By using some illustrative examples, the following issues will be demonstrated and discussed.

1. The electron multipacting looks like the dominant mechanism in pressure rise, for both gold and proton runs. The pressure rise is more sensitive to bunch spacing and bunch intensity, rather than the total beam intensity. Also, in the "intensity limiting" cases, where the beam loss effect is not dominant, the pressure rise tends to saturate for constant beam intensity, rather than to run away, indicating that the ion desorption is not a dominant factor in the RHIC pressure rise.

2. There are two types of pressure rises in the gold run. The ones caused by the beam loss have sharp rise, usually at 10 to 20 decades per minute. Since this type of rises looks like pressure run-away, it might be misidentified as the ion desorption caused pressure rise. The pressure rises caused by the electron multipacting are usually 3 to 5 decades per minute, and will reach saturation at some point.

3. For the effect of the gold ion beam loss, several pressure rise incidents have shown that the huge amount of molecules is produced at the beam loss, $1.5 \times 10^{7}$ molecules per lost gold ion. This large spattering yield has not been reported in literature, up to authors' knowledge. If this yield rate is confirmed, then small amount of gold ions lost on the chamber wall may produce enough positive ions to help electrons to survive the bunch gap, and hence support the electron multipacting.

4. For both gold and proton runs, pressure rise had only happened at the beam injection. Once the beam was accelerated, the pressure started to fall. This is explained as the result of reduced beam loss due to the reducing beam size in the acceleration, which will not produce enough positive ions to sustain the electron multipacting.

5. Intensity threshold of electron multipacting for proton run is estimated based on five 55-bunch high intensity ramps. The lowest threshold at the interaction region is about twice as high as that at either blue or yellow warm bore straight sections. Since the chambers are similar, it is speculated that the longer length of the single beam straight sections may have provided better condition for the beam halo scraping, and produced more positive ions to support the electron multipacting. Threshold of the 110-bunch gold beam at interaction is compared with the proton run. As expected, the gold beam with lower intensity, $60 \%$, has lower threshold, about $65 \%$, than the proton counterpart.
\end{abstract}




\section{Electron multipacting}

The following cases will be used to demonstrate that the pressure rise is not tightly related to the total intensity, it is, however, very sensitive to the bunch spacing. Also, for the "intensity limiting" cases, which are not directly related to the beam loss, the pressure rise will be saturated at a constant intensity, which is consistent with the electron desorption caused pressure rise, but not the one due to ion desorption [1].

Fig. 1

Ramp 1797

$11 / 19 / 01$

Intensity

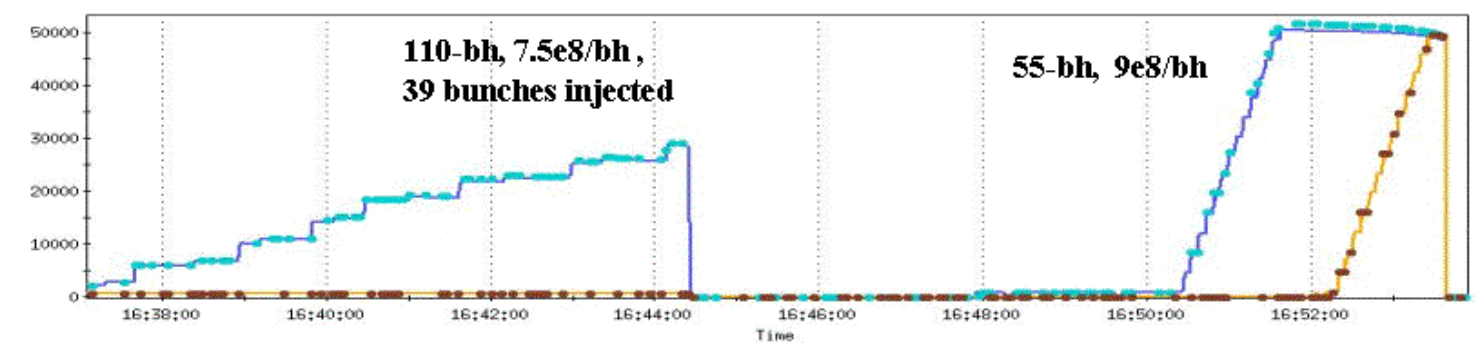

Pressure

rise

at Bo11

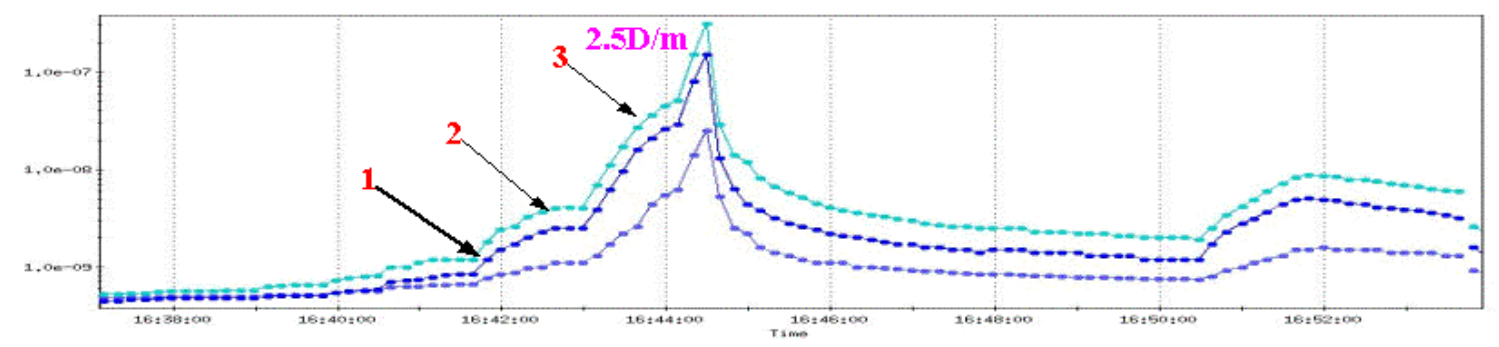

In Fig.1, the ramp 1797 beam current and the associated pressure rise at the warm straight section, Bo11, are shown. The left side is the 110-bunch injection with the bunch intensity of $7.5 \times 10^{8} A u^{79+}$ ions. After 39 bunches injected, the pressure reached $3 \times 10^{-7}$ Torr at Bo11. Vacuum valve closed at sections 3 and 4 , and the beam was aborted. The right side is the 55-bunch injection, with the bunch intensity $9 \times 10^{8}$ ions. The pressure rise was modest.

Observation and analysis are as follows.

- The 110-bunch injection, with less total intensity, caused much higher pressure rise than the 55-bunch injection. This shows that the bunch spacing is an important factor in the pressure rise. Electron multipacting looks like the dominant mechanism in the pressure rise, since the ion desorption caused pressure rise depends more on the total intensity. Ion's lifetime is in the order of $\mu s$, therefore, ion desorption caused pressure rise is less sensitive to the bunch spacing.

- Pressure rise is started at some point, for instance at $\mathbf{1}$, then it is saturated for constant beam intensity, as indicated in 2 . This is the other indication of the electron multipacting, since the electron density will be confined within the space charge limit. The ion desorption created pressure rise is usually with run-away type above the threshold, and sometimes it is called 'pressure instability'.

- The pressure rise saturation level, hence the electron density, is not linearly proportional to the intensity. As shown in 3 , the pressure saturation level is increased by an order from the point 2 , whereas the intensity is increased about 10\%. Similar phenomenon has been observed at the PEP II [2]. This fact cannot yet be explained by the theory and simulation [3], where the electron density is predicted to be linearly proportional to the bunch intensity averaged over the bunch spacing. More study is needed.

- In this ramp, the largest pressure rise rate of the 110-bunch injection, before the valve close, is $2.5 \mathrm{D} / \mathrm{m}$, i.e. two and half decades per minute. This rate will be compared later with the one caused by beam loss. 
Fig. 2

Ramp1795, 11/19/01
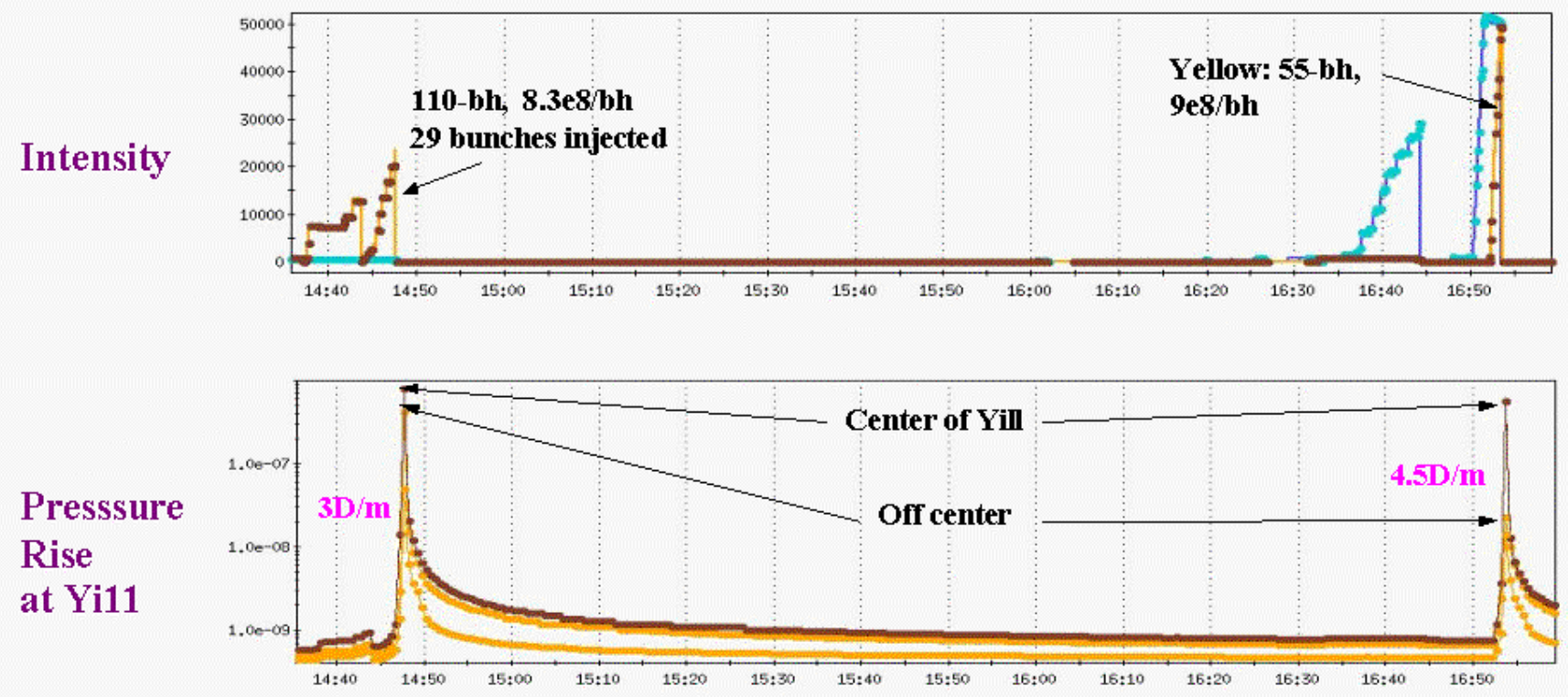

In Fig.2, the pressure rises at Yi11 are shown for the ramps 1795 and 1797, separated by about 2 hours. Again, for 110-bunch injection with lower intensity, the peak pressure rise is about equal to the pressure rise of the 55-bunch injection.

Fig. 3

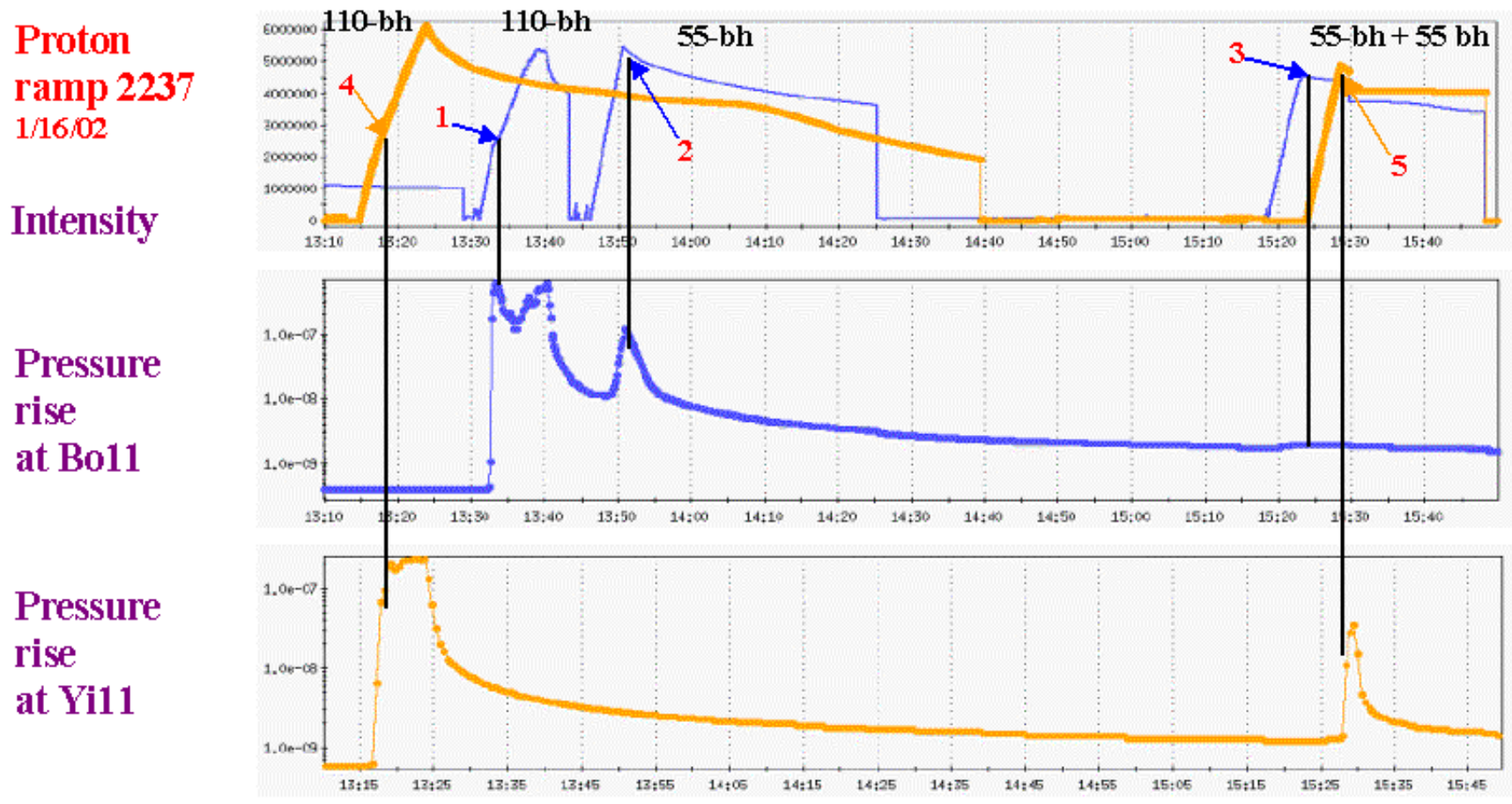


In Fig.3, the pressure rises at Bo11 and Yi11 are shown for 110-bunch and 55-bunch injections in the proton ramp 2237.

In this ramp, the 110-bunch injection was tampered by the imperfect functioning of injection kickers. As a result, the 110-bunch injection gave rise to almost same total intensity of the 55-bunch injection. However, same evidence of the electron multipacting caused pressure rise is still presented.

- At Bo11, the 110-bunch injection with the total intensity of $24 \times 10^{11}$ protons, shown at 1 , gives rise to higher pressure rise than the 55-bunch injection with the total intensity of $55 \times 10^{11}$ protons, shown at 2 .

- The pressure rise corresponding to 2 is probably aggravated by the leftover pressure. Another 55-bunch injection with comparable intensity, shown at 3 , caused very little pressure rise.

- For Yil1, the pressure rise of 110-bunch intensity, shown at 4, is also higher than the one caused by the higher intensity of the following 55-bunch injection, shown at 5 .

\section{Two types of pressure rise in gold run}

Many pressure rise incidents in the gold run are caused by the beam loss. Several cases are completely due to the electron multipacting, and identified as the "intensity limiting" cases. In these cases, the pressure rise rate is usually 3 to 5 decades per minute, and the pressure will reach saturation at some point. On the other hand, the beam loss caused pressure rises have sharp rising slope, usually at 10 to 20 decades per minute, and look like pressure run-away. Note that the run-away type of pressure rise might be misidentified as the ion desorption caused pressure rise.

\section{Fig. 4}
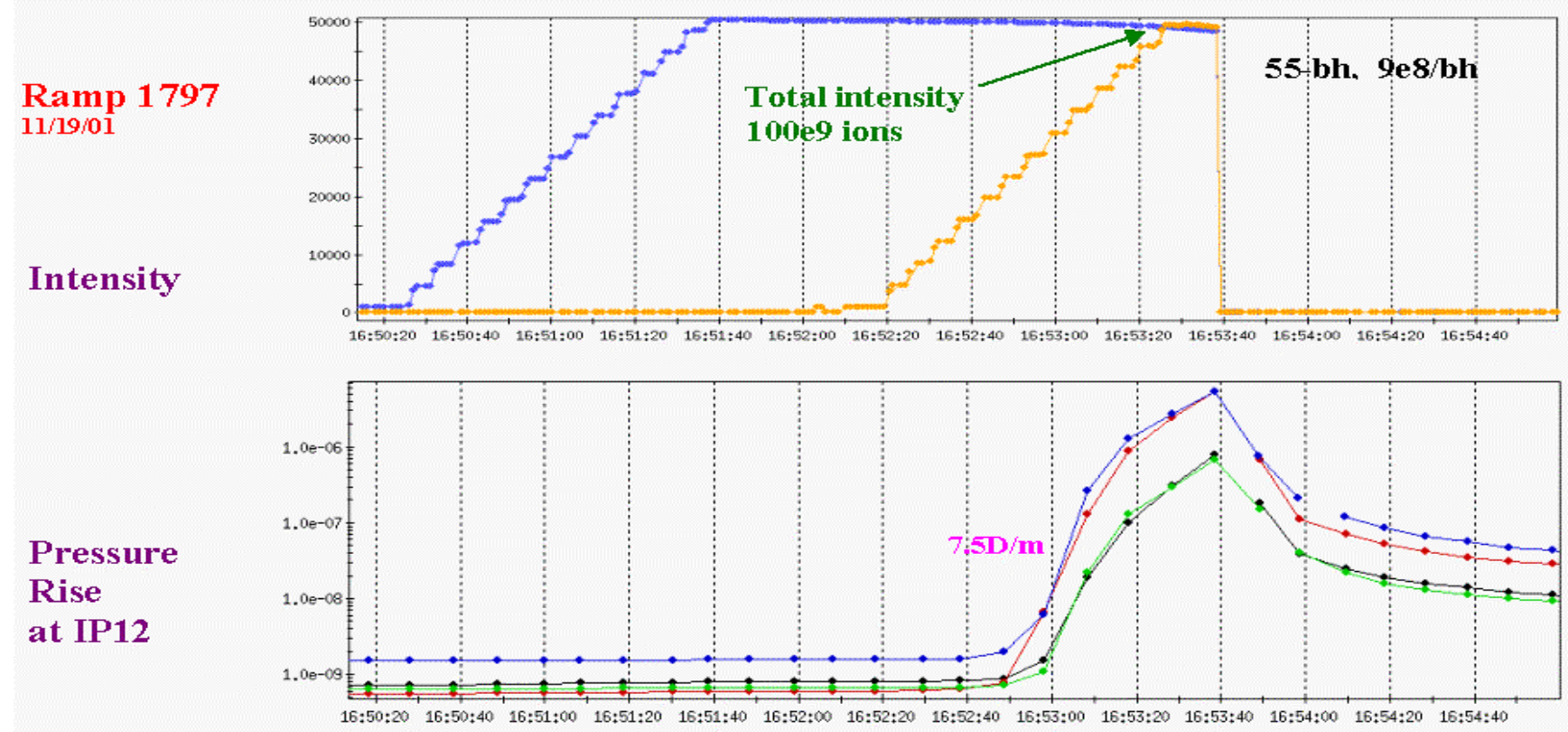

In Fig.4, an "intensity limiting" type of pressure rise at IP12 is shown. The pressure rise is started at a threshold, then approaches saturation. The pressure rise rate at IP12,7.5D/m, is higher than a typical electron multipacting case, it is probably because of the high bunch intensity, $0.9 \times 10^{9}$ ions, and/or associated beam losses.

The total peak intensity at IP12 is about $100 \times 10^{9}$ ions, which is the highest total intensity in the gold beam 55 -bunch fillings, no sign of run-away type pressure rise is shown. 


\section{Fig. 5}

Ramp 1357

10/17/01

Intensity
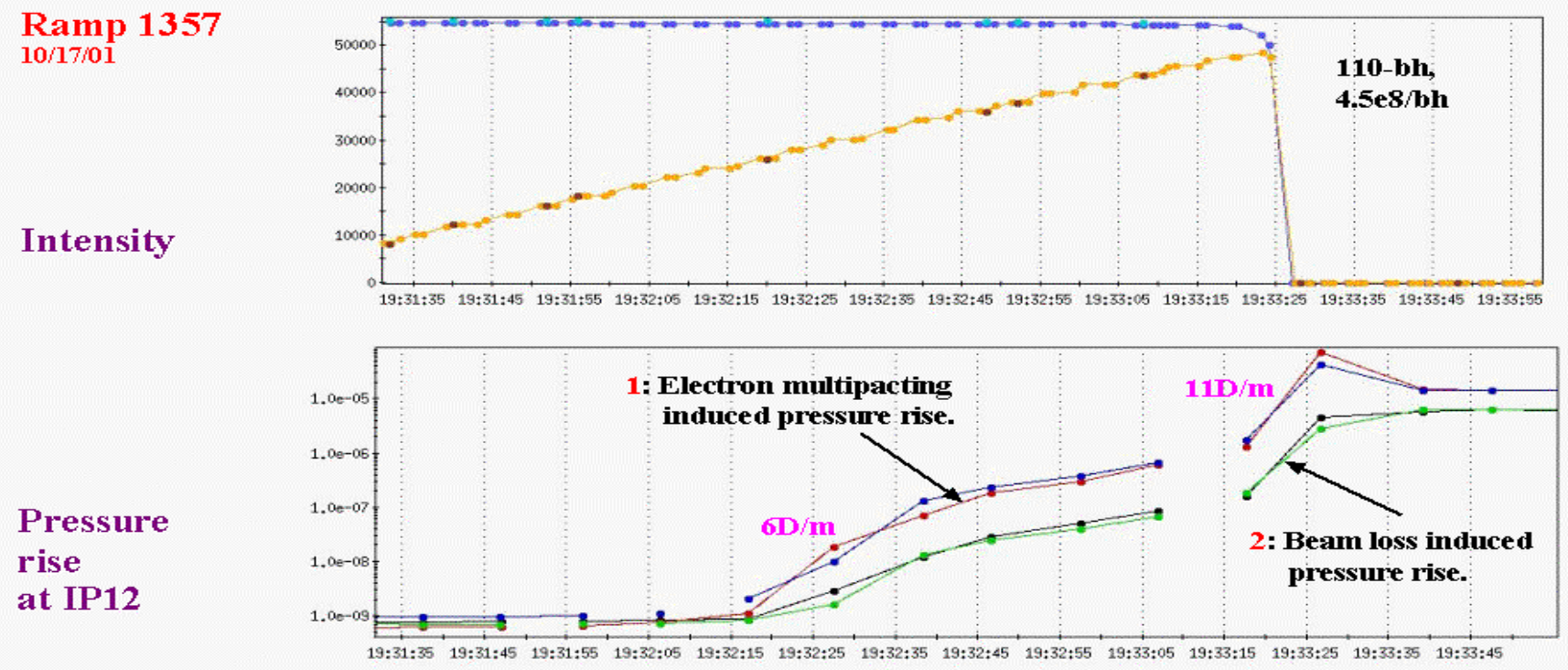

Fig. 5 shows a rare case of the electron multipacting, which is followed by a sudden beam loss. This was a 110-bunch injection with medium bunch intensity of $4.5 \times 10^{8}$ ions. Note that the electron multipacting caused pressure rise was approaching saturation, even the yellow beam filling was not completed, shown at 1 , where the highest pressure rise slope is $6 \mathrm{D} / \mathrm{M}$. Once the beam loss happened, the slope became larger than $11 \mathrm{D} / \mathrm{m}$, shown at 2 .

Fig. 6

Ramp 1830

$11 / 22,01$

Intensity

Pressure

rise

at IP2

Pressure
rise
at IP10
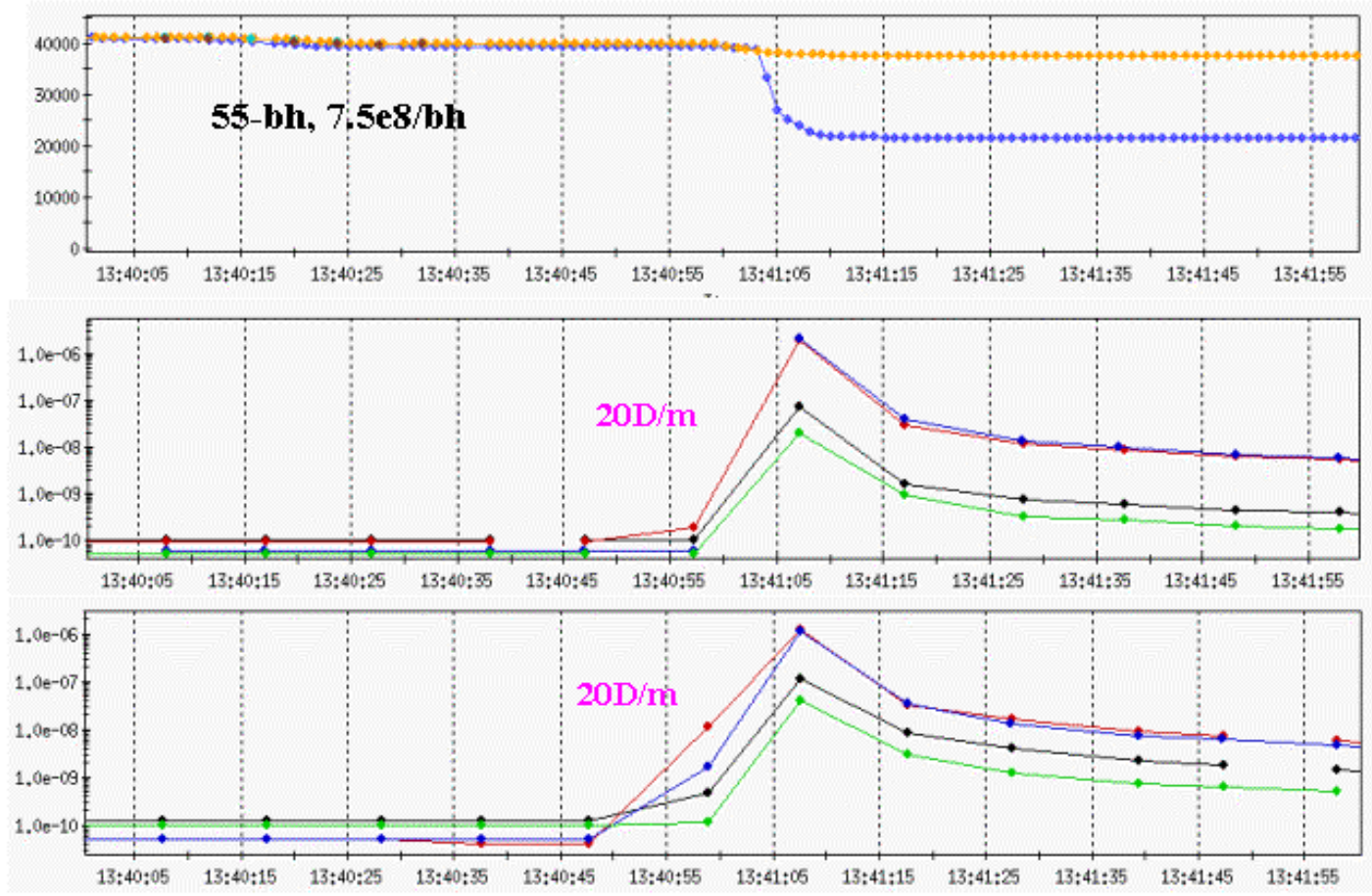

Fig. 6 shows a case that only the blue ring lost half of the beam, pressure rises occur at IP2 and IP10 to above $10^{-6}$ Torr, in run-away type. 


\section{Gold ion beam loss effect}

The following cases, together with the one shown in Fig.5, will show that the gold ion beam loss might be able to desorb huge number of molecules.

\section{Fig. 7}

Ramp 1539

10/29/01

Intensity

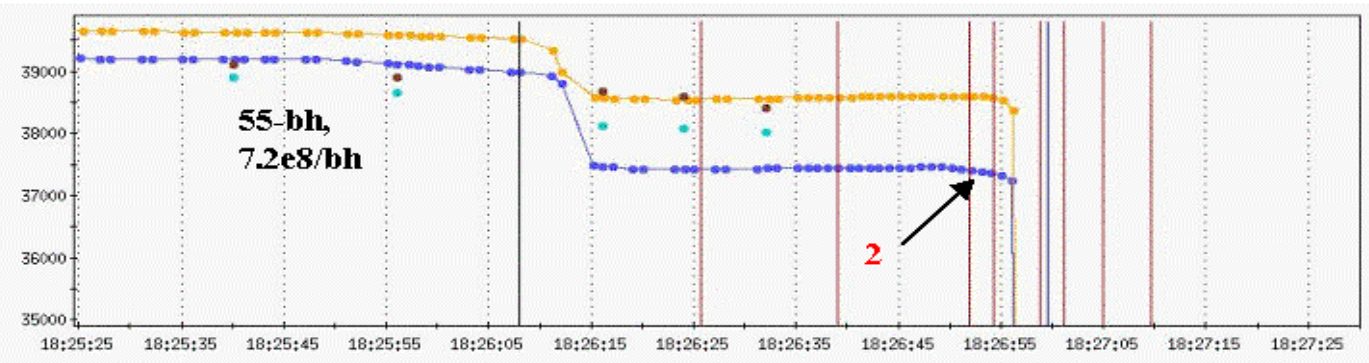

Pressure

rise

at IP12

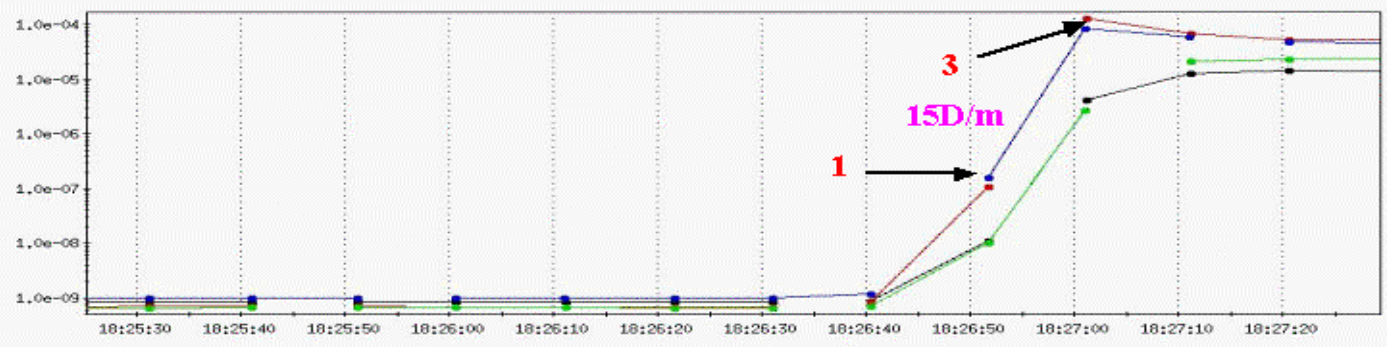

A beam loss induced pressure rise at IP12 is shown in Fig.7.

- Taking IP12 as 30 meters long, with $6 \mathrm{~cm}$ average pipe radius, total volume is $0.34 \mathrm{~m}^{3}$. At 1 , pressure is higher than $10^{-7}$ Torr. Using the beam loss of $2.5 \times 10^{9}$ happened about 40 seconds earlier, it is calculated that every lost ion created $5 \times 10^{5}$ molecules. However, this pressure rise might be caused by much smaller beam loss taking place at that moment, shown at 2, then much higher spattering yield can be expected.

- At 3, the pressure reached 10-4 Torr. Assuming all $79 \times 10^{9}$ ions lost at IP12, then each ion has desorbed $1.6 \times 10^{7}$ molecules. On the other hand, the spattering yield reported in literature, for instance in [4], is from 0.1 to $10^{6}$.

Fig. 8 shows the pressure rises caused by beam loss at IP12 and IP2, for the ramp 1754 ..

- The peak pressure caused by the beam loss at IP12 is $10^{-4}$ Torr, at IP2 it is $3 \times 10^{-6}$ Torr. Pressure rises also observed as $1.5 \times 10^{-7}$ Torr at IP10, and as $5 \times 10^{-8}$ Torr at IP4. On the other hand, none of the warm straight sections had pressure rise.

- For the pressure rise of 10-4 Torr at IP12, assuming all $80 \times 10^{9}$ gold ions lost there and without significant pumping, then every lost ion has desorbed $1.5 \times 10^{7}$ molecules.

- Note that the pressure rise rates are $12 \mathrm{D} / \mathrm{m}$ and $24 \mathrm{D} / \mathrm{m}$, for IP12 and IP2, respectively.

- Since that no significant beam loss can be identified in the middle of the pressure rise at IP12, one may question if the run-away type pressure rise is caused by the ion desorption. Note the high intensity 55bunch injection shown in Fig.4, where the total intensity had reached $100 \times 10^{9}$ ions, the pressure rise was still approaching saturation. Also, the 110-bunch injection shown in Fig.5 reached the same total intensity of $100 \times 10^{9}$ ions, the run-away type pressure rise did not show up, until the beam loss. Therefore, the chance that ion desorption contributed to the pressure rise at IP12 is slim. 


\section{Fig. 8}

\section{Ramp 1754 \\ $11 / 14 / 01$}

\section{Intensity}
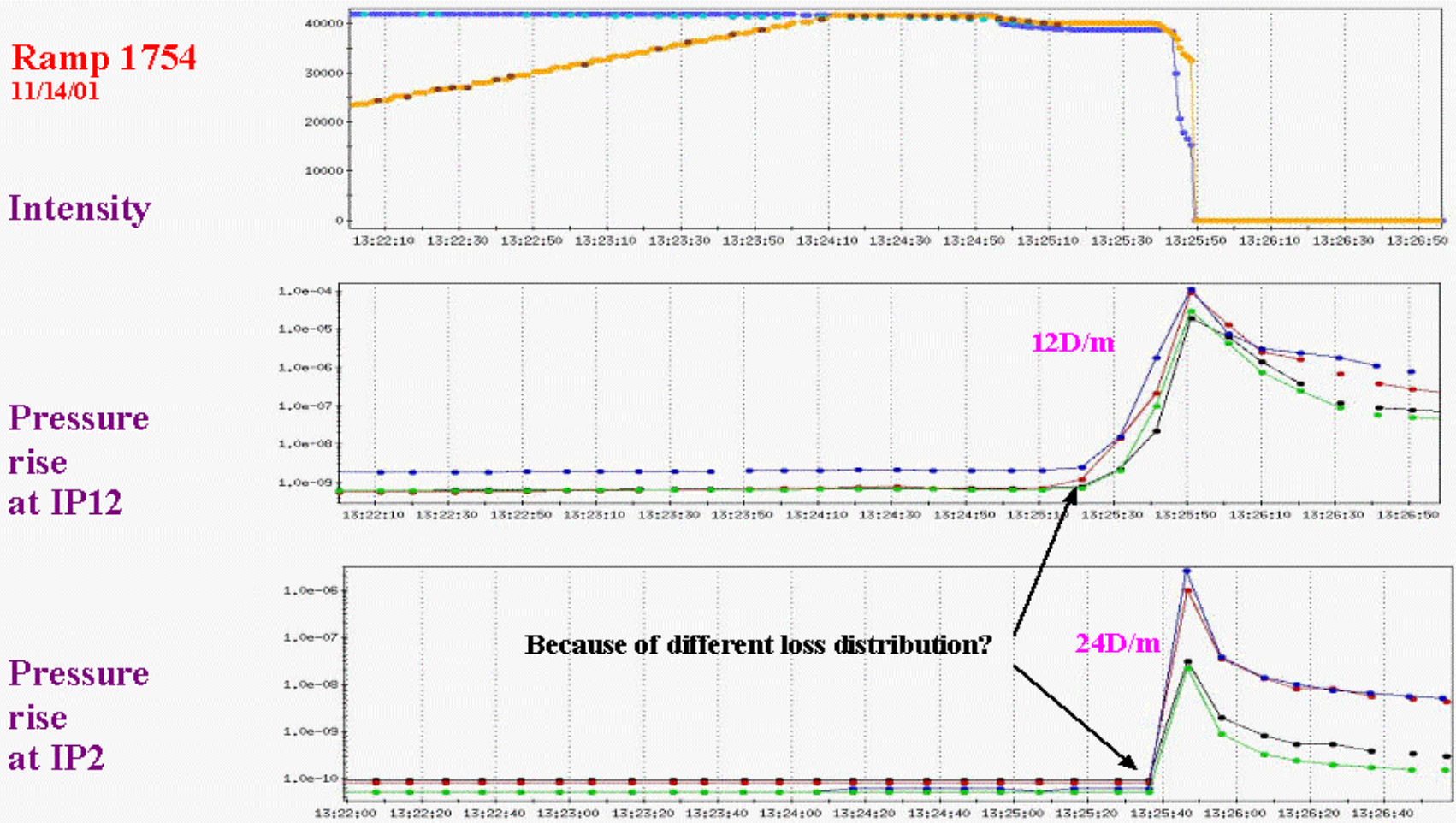

In Fig.9, the pressure rise at IP12 for the ramp 1169 is shown, which reached $2 \times 10^{-4}$ Torr, the highest in the run. Afterwards, the vacuum pumping stopped functioning in about 10 minutes. Total beam intensity was about $70 \times 10^{9}$ ions, much less than the one in Fig.5.

Fig. 9

Ramp 1169

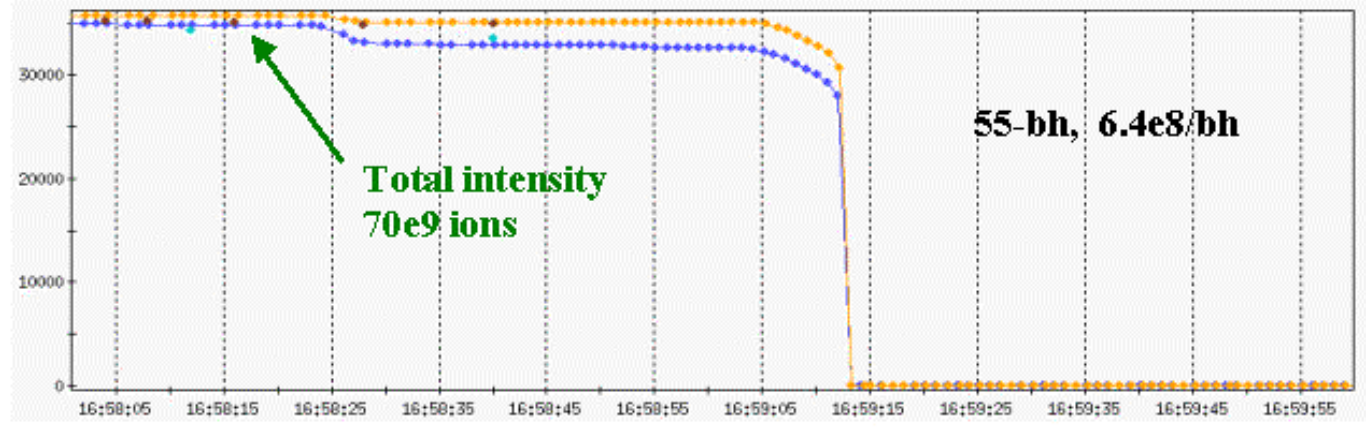

Pressure

rise

at IP12

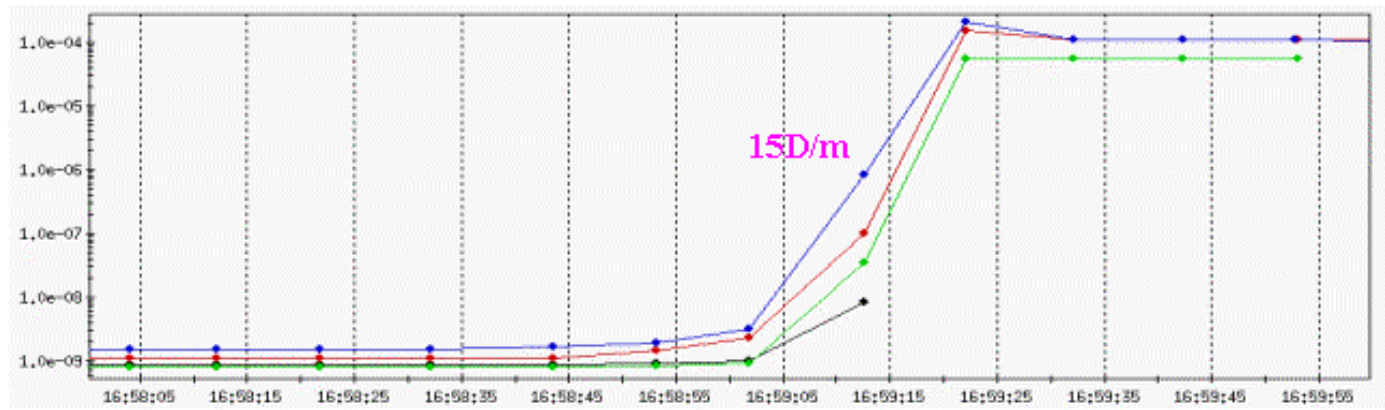


In Table 1, some of run-away pressure rises associated with the beam loss are shown. The time period covers from early September to later November.

Table 1: Examples of run-away type pressure rises associated with beam loss

\begin{tabular}{|c|c|c|c|c|c|c|}
\hline Ramp, Date & $\begin{array}{c}\text { Injection } \\
\text { Pattern } \\
\end{array}$ & $\begin{array}{c}\text { Lost ions } \\
10^{9} \\
\end{array}$ & $\begin{array}{c}\text { IP2 } \\
\text { Press. Torr }\end{array}$ & $\begin{array}{c}\text { IP4 } \\
\text { Press. Torr }\end{array}$ & $\begin{array}{c}\text { IP10 } \\
\text { Press. Torr }\end{array}$ & $\begin{array}{c}\text { IP12 } \\
\text { Press. Torr }\end{array}$ \\
\hline $0951,09 / 10 / 01$ & 6-bunch & 6 & & $1.5 \mathrm{e}-5$ & & \\
\hline $1080,09 / 24 / 01$ & 55-bunch & 60 & $1 \mathrm{e}-5$ & & & \\
\hline $1169,10 / 02 / 01$ & 55-bunch & 68 & $1 \mathrm{e}-5$ & & & $2 \mathrm{e}-4$ \\
\hline $1477,10 / 25 / 01$ & 55-bunch & 74 & $2 \mathrm{e}-5$ & & & $1 \mathrm{e}-6$ \\
\hline $1547,10 / 30 / 01$ & 55-bunch & 76 & $2 \mathrm{e}-6$ & & & 8 e-7 \\
\hline $1754,11 / 14 / 01$ & 55-bunch & 80 & $2 \mathrm{e}-6$ & $5 \mathrm{e}-8$ & $1.5 \mathrm{e}-7$ & $1 \mathrm{e}-4$ \\
\hline $1830,11 / 22 / 01$ & 55-bunch & 20 & 2 e-6 & & $1 \mathrm{e}-6$ & $2.5 \mathrm{e}-7$ \\
\hline
\end{tabular}

\section{Beam loss and electron multipacting}

For both gold and proton runs, no vacuum pressure rise has been observed at the storage. In this section, several cases will be used to show that once the beam is accelerated, the pressure starts to fall. This may indicate that the beam loss indeed aggravated the electron multipacting in the RHIC.

Fig. 10

\section{Proton \\ Ramp 2243 \\ $1 / 16 / 02$}

Intensity

Pressure

rise

at IP12

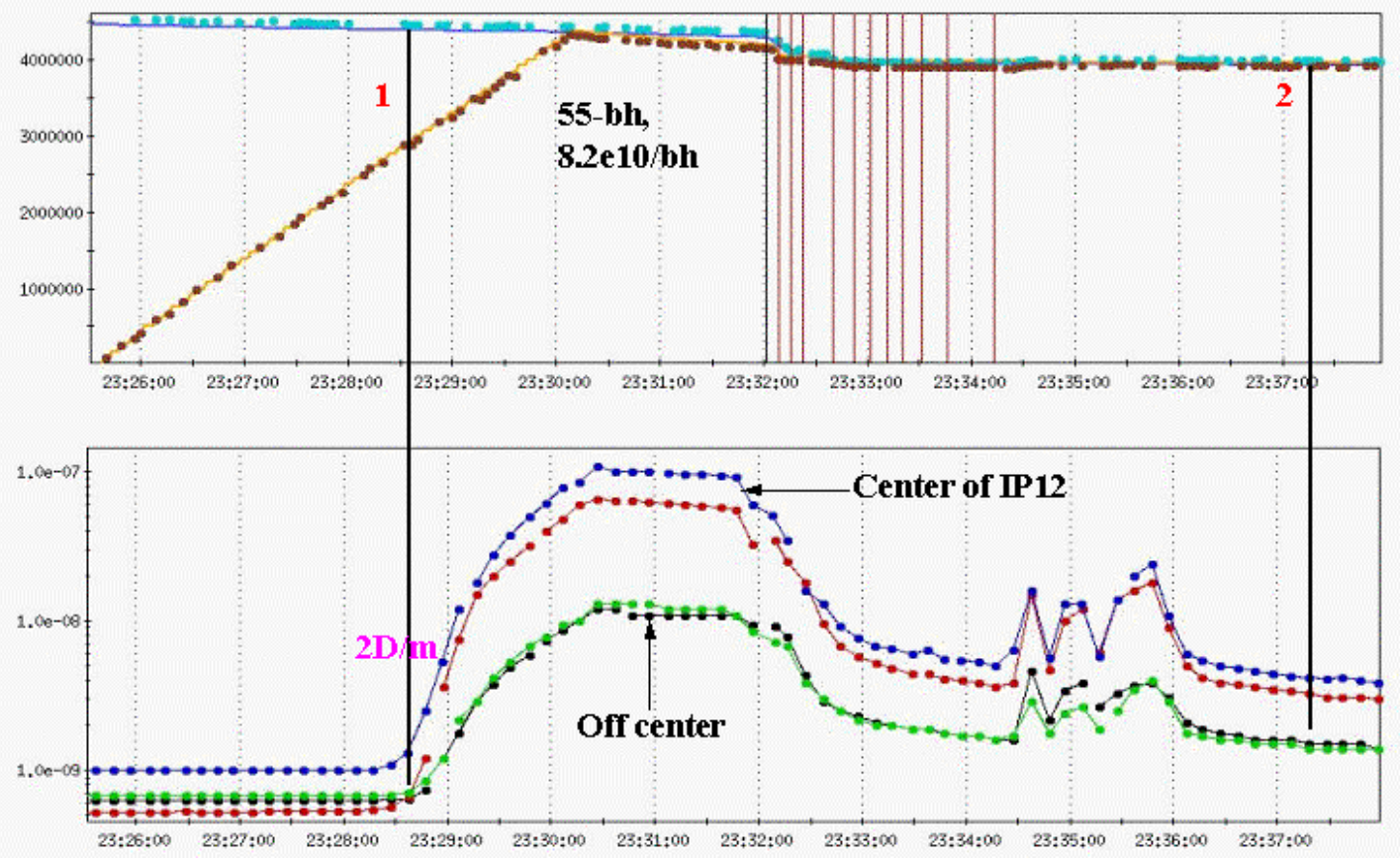


In Fig.10, the proton ramp 2243 is used to demonstrate that once the beam is accelerated, the pressure rise is disappeared, this is observed for most pressure rising cases in the proton run.

- This ramp shows a typical pressure rise pattern in the proton run, which carries several characteristics:

1. Once a threshold is reached, the pressure starts to rise, then it will be saturated. This pattern is in agreement with the pressure rise caused by electron multipacting.

2. The highest pressure in proton run is usually in $10^{-7}$ Torr level. The valves were never closed during the proton operation and machine studies. No run-away type of pressure rise, like the ones caused by the beam loss in the gold run, has been observed.

3. Once the beam is accelerated, the pressure starts to fall.

4. The pressure glitch at the early store might be due to orbit steering [5].

- Total beam intensity at $\mathbf{2}$ is higher than the one at the electron multipacting threshold, shown at $\mathbf{1}$, but the pressure is falling at 2 . A possible reason is that in the beam acceleration, the transverse beam size is reduced rapidly. As the result, the beam halo scraping on the chamber wall will be reduced. Once there is not enough beam loss produced positive ions to support the electron multipacting, pressure rise cannot sustain.

- Another factor is that the average bunch spacing at the interaction region may become longer at the beam acceleration $[5,6]$. The contribution of this factor is not very clear, but worth some attentions.

\section{Fig. 11}

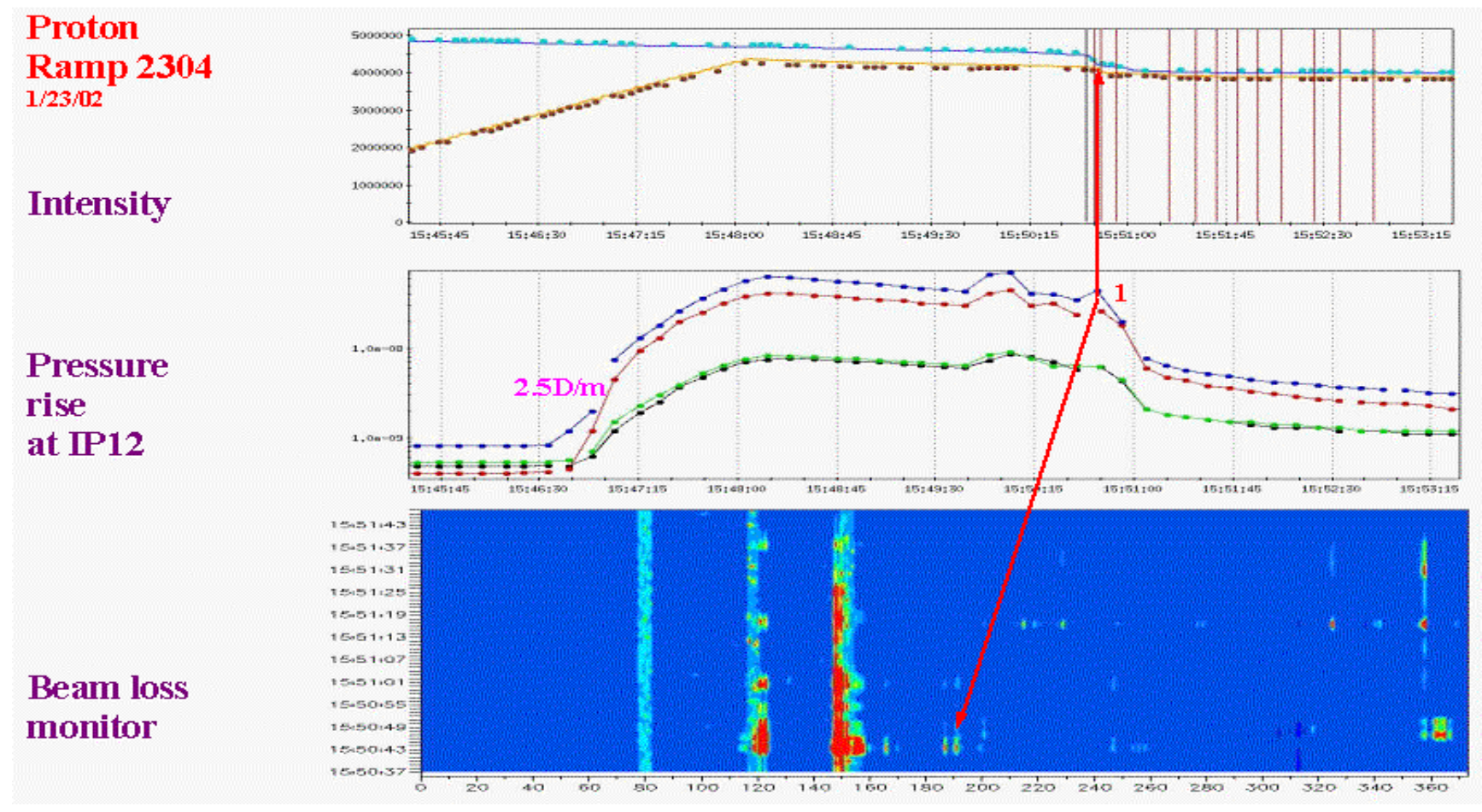

In Fig.11, the ramp 2304 also shows that the pressure falls as the beam is accelerated. In this case, the small pressure rise at 1 is related with the beam loss, as shown at the DCCT beam intensity and the beam loss monitor, at IP12. 


\section{Fig. 12}

\section{Proton}

Ramps 2189 - 2194 $1 / 10 / 02$

\section{Intensity}

55 bunch

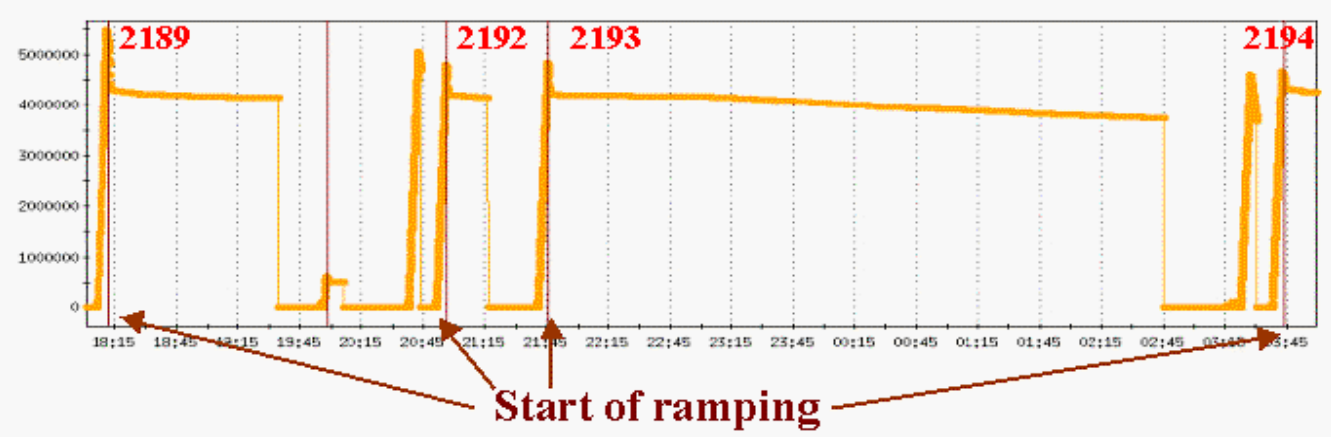

\section{Pressure}

rise

at Yi11

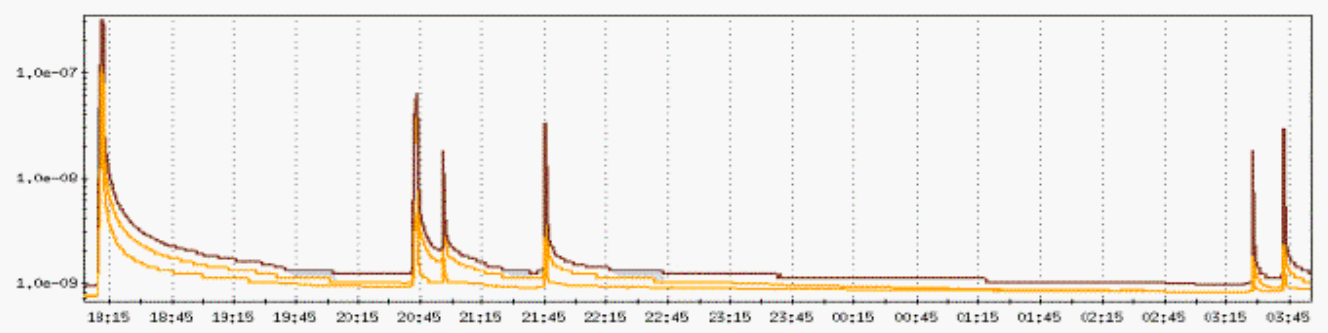

In Fig.12, the pressure rise at Yil1 are shown for the proton ramps 2189 to 2194. The injected bunch intensity is around $10^{11}$, whereas the stored bunch intensity is a little lower than $0.8 \times 10^{11}$. Note the pressures are all falling at the acceleration. Since there is only one beam at Yil1, therefore, the bunch spacing is unchanged as the beam is accelerated.

\section{Fig. 13}

\section{Proton}

Ramp 2158

$1 / 06 / 02$

\section{Intensity}

55 bunch

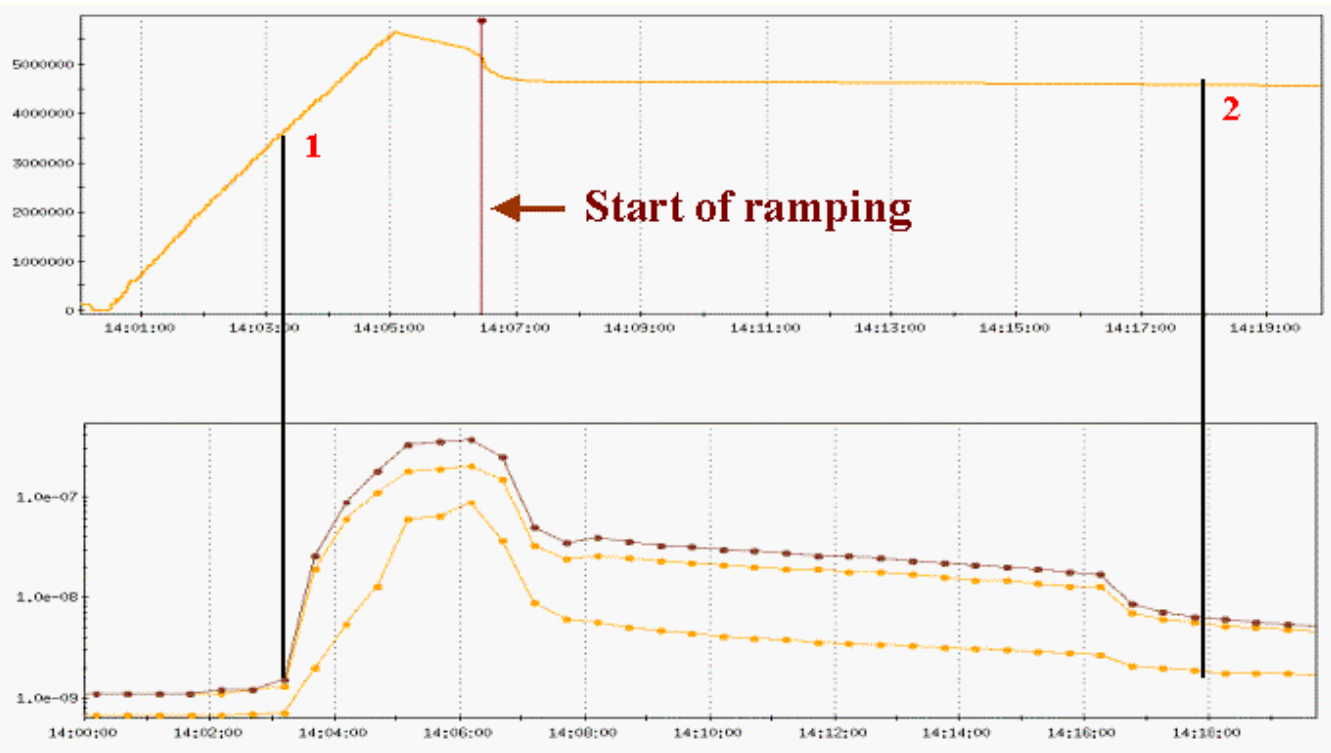

In Fig.13, the close look of pressure rise at Yil1 is shown for the ramp 2158. The pressure falls as the beam is accelerated. The total intensity at store is $46 \times 10^{11}$, shown at 2 , which is higher than the intensity threshold of the electron multipacting, $35 \times 10^{11}$, at 1 . 


\section{Electron multipacting threshold}

In the gold run, there are only limited "intensity limiting" cases. Therefore, the proton run will be reviewed for estimating electron multipacting threshold first, then some cases in the gold run will be compared.

Fig. 14

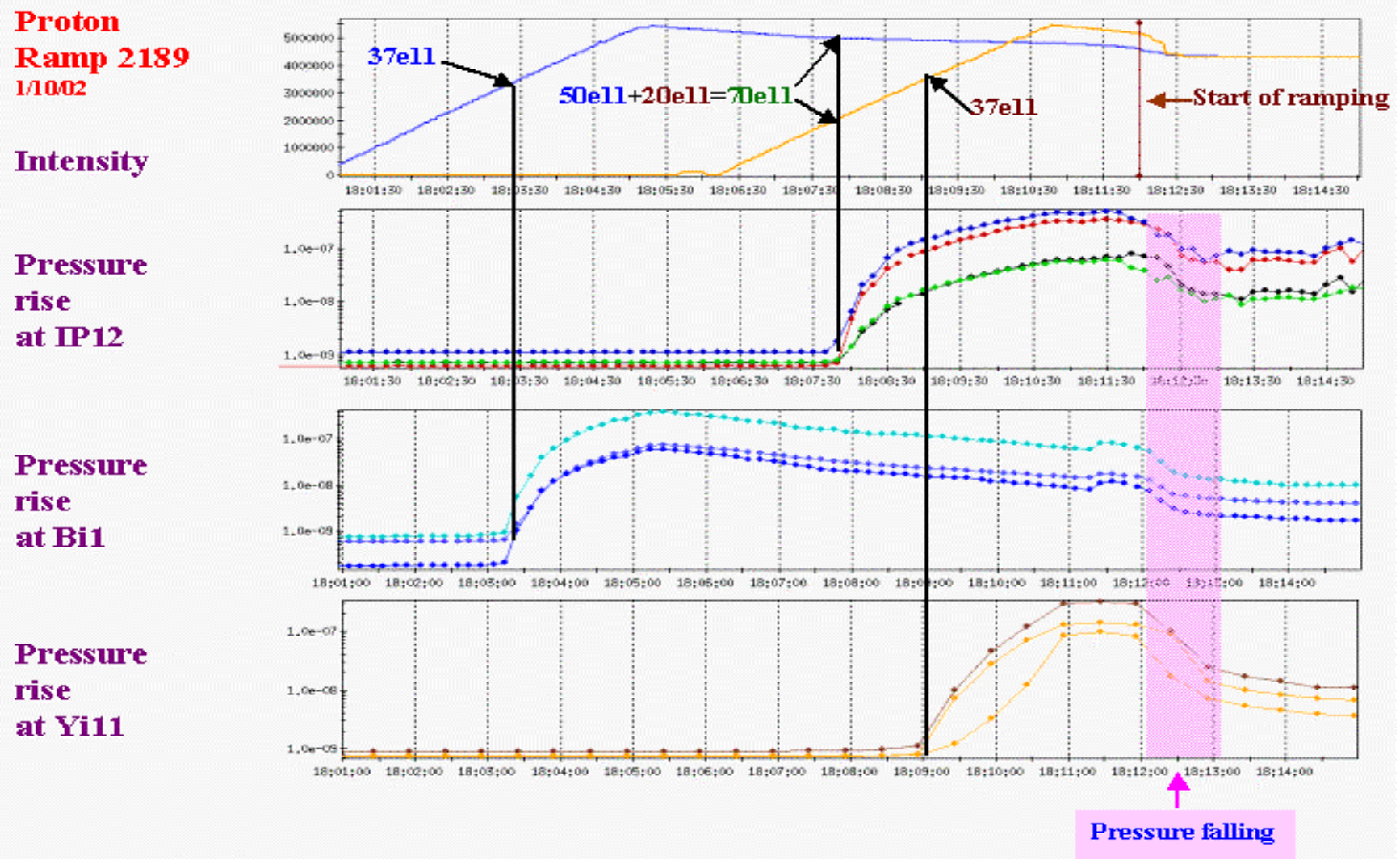

In the proton ramp 2189, shown in Fig.14, the high pressure rises at IP12, Bil and Yi11 are used to estimate the electron multipacting threshold for interaction region, blue and yellow, respectively. Thresholds are indicated at 1, 2, and 3, for each type of the pipes. Beam scrubbing might be taking effect at Bil before the acceleration. The pressure rise pattern at Yi11 is similar to that shown in Fig. 13 for the ramp 2158.

Note that as the ramp started, the pressure started to fall at all places, IP12, Bil and Yi11.

The thresholds of five 55-bunch proton ramps, including the ramp 2189, and one 110-bunch ramp, are shown in Table 2.

\section{Table 2: Electron multipacting threshold}

\begin{tabular}{|c|c|c|c|c|c|}
\hline Ramp & Inj. pattern & Bunch Int. $10^{11}, \mathrm{~B} / \mathrm{Y}$ & $\mathbf{I P 1 2}, 10^{11}, \mathrm{~B}+\mathrm{Y}=$ Total & ${\text { Bi1, } 10^{11}}^{1}$ & ${\text { Yi11, } 10^{11}}^{11}$ \\
\hline 2158 & 55 -bunch & $1.1 / 1.05$ & $50+20=70$ & 40 & 35 \\
\hline 2170 & 55 -bunch & $0.95 / 0.95$ & $50+27=77$ & 48 & 40 \\
\hline 2189 & 55 -bunch & $1.0 / 1.0$ & $50+20=70$ & 37 & 37 \\
\hline 2196 & 55 -bunch & $0.9 / 0.92$ & $43+33=76$ & 46 & 46 \\
\hline 2208 & 55 -bunch & $1.0 / 0.85$ & $47+27=74$ & 34 & 42 \\
\hline Average & $\mathbf{5 5 - b u n c h}$ & $\mathbf{1 . 0 / 0 . 9 5}$ & $\mathbf{7 3 . 4}$ & $\mathbf{4 1}$ & $\mathbf{4 0}$ \\
\hline $\mathbf{2 2 3 7}$ & $\mathbf{1 1 0}$-bunch & $\mathbf{1 . 0} / \mathbf{1 . 0 5}$ & N/A & $\mathbf{1 8}$ & $\mathbf{2 0}$ \\
\hline
\end{tabular}




\section{comparison.}

In all these cases, the bunch intensities are about $10^{11}$, which provides convenience in the threshold

- IP12 usually has the lowest threshold among the interaction sections, however, its threshold is almost twice as high as either Bi1, or Yi11.

- Chambers at these locations are all stainless steel, unbaked, with same radius. Average bunch spacing at IP12 is shorter than Bil and Yi11, which is actually in favor of the electron multipacting.

- It is noted, however, the length of the straight vacuum pipes at Bi1 and Yi11 are longer than IP12. One possibility is that the beam halo scraping at longer straight pipes has produced more positive ions, which aggravated the electron multipacting. There are other issues, such as the beam size, aperture, ..., which may be taking effect.

- Recall that the vacuum chambers and conditions at IP12 and IP4 are very similar, but IP4 was much less troublesome with respect to the pressure rise in the gold run. It was speculated in [1] that many chamber interruptions at IP4 may have prevented effective beam scraping, and hence have increased electron multipacting threshold.

- For blue and yellow warm bore straight sections, the threshold of 110-bunch injection is about half of that of the 55-bunch injection.

In the following, Fig.15 shows the proton beam, and Fig.16 shows the gold beam 110-bunch injections. The pressure rises at Bil and Yi11 are used to compare the electron multipacting threshold.

\section{Fig. 15}

\section{Proton}

Ramp 2237

$1 / 1602$

Intensity, Blue

Pressure

rise

at Bi1

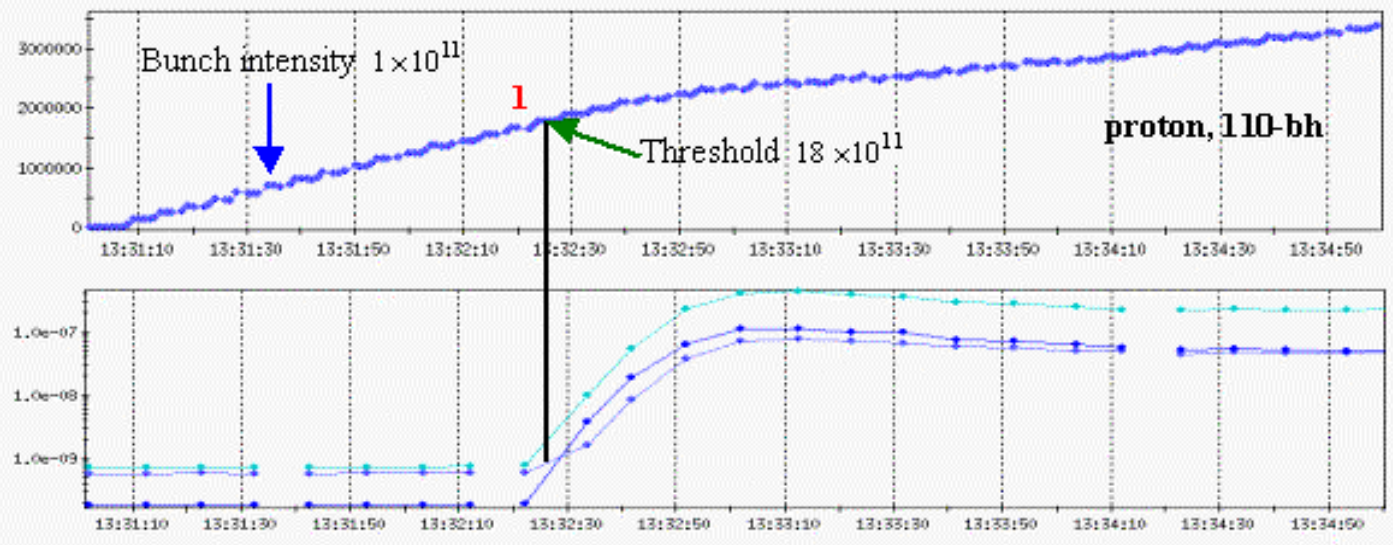

Intensity, Yellow

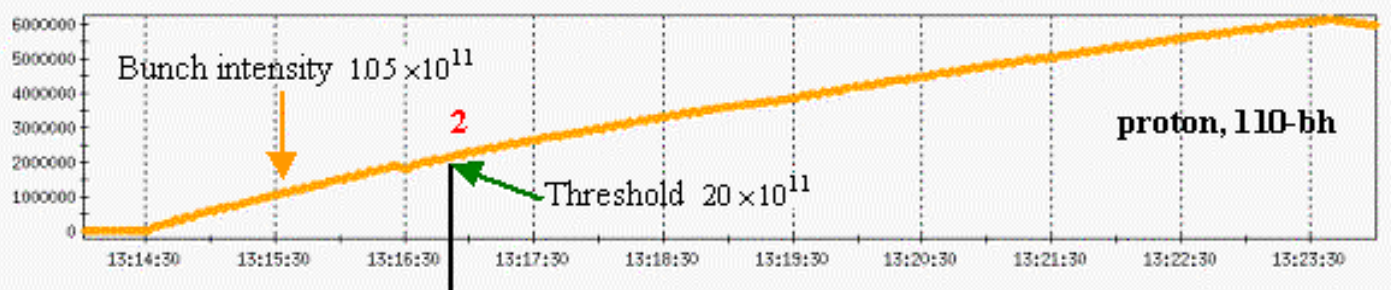

\section{Pressure}

rise

at Yi11

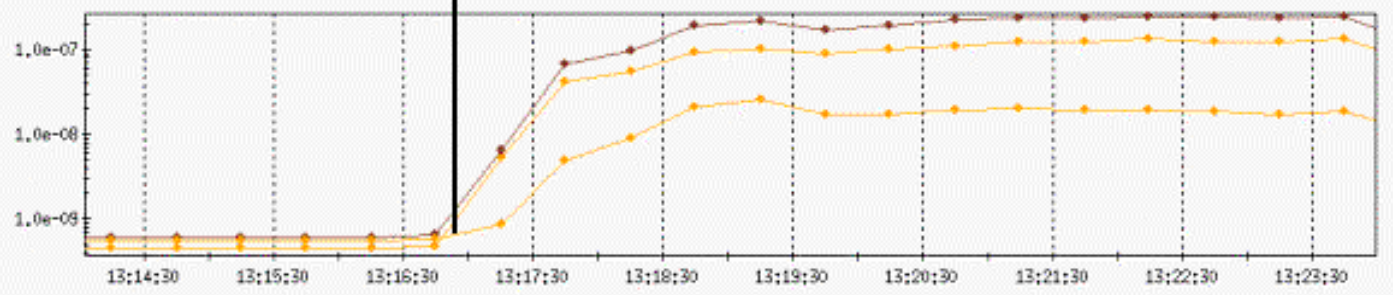


Fig. 16

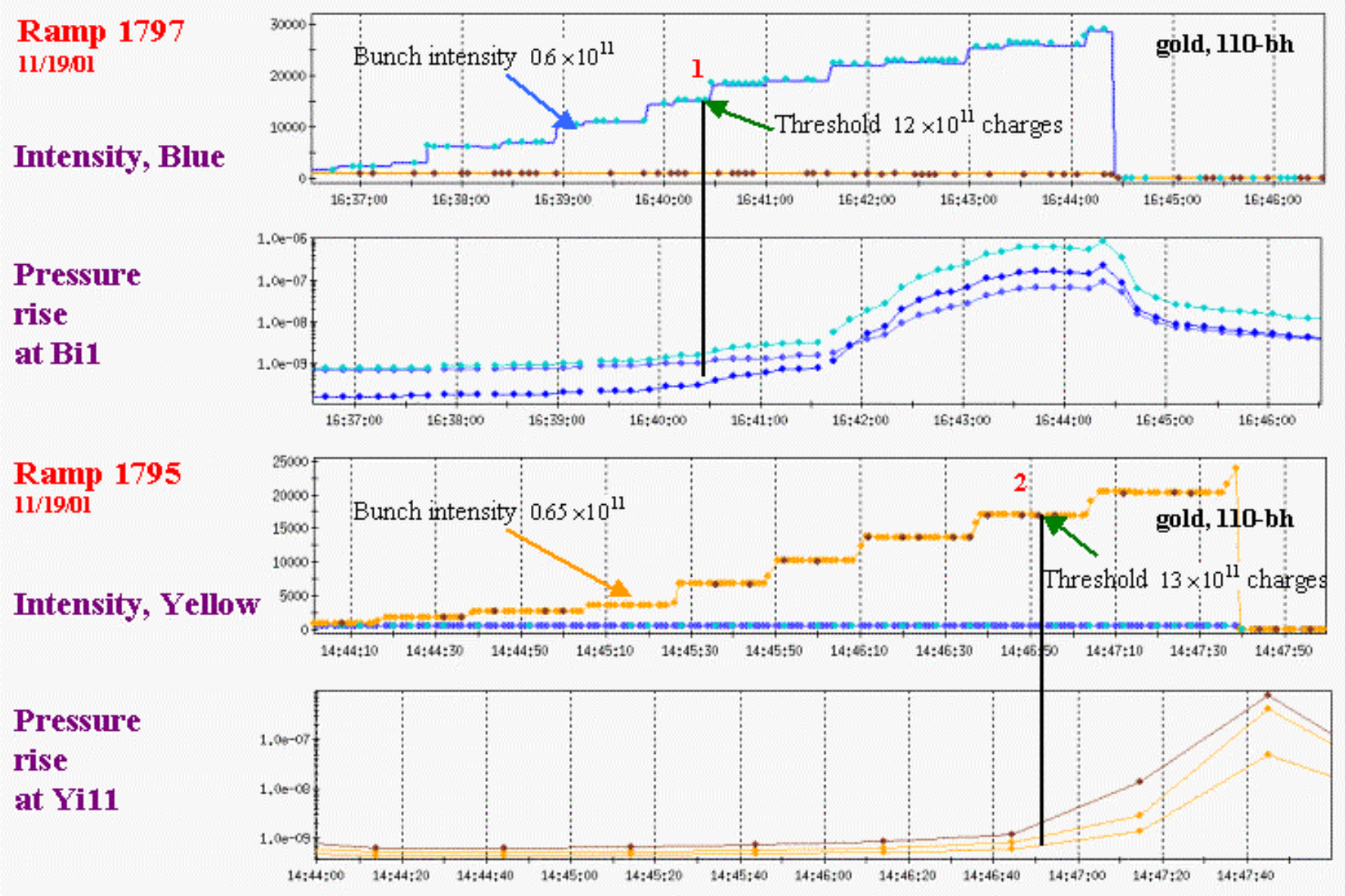

In Table 3, a comparison of proton and gold beam thresholds is given.

Table 3: Proton and gold thresholds for 110 bunches

\begin{tabular}{|c|c|c|c|c|}
\hline & $\begin{array}{c}\text { Bunch charge, } \\
10^{11}, \text { Blue }\end{array}$ & $\begin{array}{c}\text { Bunch charge, } \\
10^{11}, \text { Yellow }\end{array}$ & $\begin{array}{c}\text { Bi1 } \\
10^{11}\end{array}$ & $\begin{array}{c}\text { Yi11 } \\
10^{11}\end{array}$ \\
\hline Gold, 110-bunch & $\mathbf{0 . 6}$ & $\mathbf{0 . 6 5}$ & $\mathbf{1 2}$ & $\mathbf{1 3}$ \\
\hline Proton, 110-bunch & $\mathbf{1 . 0}$ & $\mathbf{1 . 0 5}$ & $\mathbf{1 8}$ & $\mathbf{2 0}$ \\
\hline
\end{tabular}

The gold beam charges are about $60 \%$ of the proton's, however, the thresholds are lower, at about $65 \%$ of the proton's. 


\section{Acknowledgment}

We would like to thank W. Fisher, W. MacKay, V. Ptitsyn, S. Peggs, T. Roser, D. Trbojevic, J. Wei for helpful discussions. We also acknowledge that several very useful cases, especially the 110-bunch fills, were set up in the dedicated RHIC machine study.

\section{References}

1. S.Y. Zhang, C-AD AP//67, Jan. 2002.

2. S. Heifets, Two-Stream Workshop, LANL, Feb. 2000.

3. F. Zimmermann, Two-Stream Workshops, LANL, Feb. 2000 and KEK, Sep. 2001.

4. "Spattering by Particle Bombardment", Edited by R. Behrisch, Springer-Verlag, New York, 1981.

5. V. Ptitsyn, private communication.

6. W. MacKay, private communication. 